\title{
Comparative evaluation of effects of subgingival application of ozonated olive oil versus chlorhexidine in patients with chronic periodontitis: A clinico- microbiological randomised trial
}

\author{
Medhavee Khare ${ }^{1, *}$, Kavya Sangal Jain ${ }^{2}$, Nikhil Sharma ${ }^{3}$ \\ ${ }^{1,2}$ Post Graduate Student, ${ }^{3} \mathrm{HOD}$, Dept. of Periodontology and Implantology, I.T.S Centre for Dental Studies \& Research, Muradnagar, \\ Ghaziabad, India
}

*Corresponding Author: Medhavee Khare

Email: medhaveekhare.cancer@gmail.com

\begin{abstract}
Introduction: Chronic periodontitis is an infectious disease resulting in inflammation within the supporting tissues of the teeth, progressive attachment loss and bone loss. Non-surgical periodontal treatment of chronic periodontitis poses a great challenge. Conventional treatment includes Scaling and Root Planing (SRP) and mechanical debridement. Studies have suggested use of ozonated olive oil for management of periodontal infection.

Objective: The aim of this study was to evaluate the effect of using Ozonated olive oil along with SRP, compared to subgingival chlorhexidine with SRP for treatment of chronic periodontitis.

Materials and Methods: In this split-mouth design, twenty untreated chronic periodontitis patients who met the criteria of having periodontal pocket with depth $\geq 4 \mathrm{~mm}$ and bleeding on probing (BOP) in at least two different quadrants were included. Group 1 (control) subgingival chlorhexidine application was done and in group 2 (test) ozonated olive oil application with SRP was done. Clinical parameters were evaluated at baseline, 7 days, 14 days and 21 days post treatment and plaque samples at baseline and 21 days. Microbiological analysis was done to assess the change in level of P. gingivalis.

Result: Both the groups (test \& control) showed improvement in all the recorded parameters (PI, GI, PPD\& microbiological analysis) while the test group showed statistically significant reduction in all the clinical parameters compared to control group ( $\mathrm{p}<0.05)$ except the microbiological analysis.

Conclusion: A single subgingival irrigation with ozonated oil can be effectively used as an adjunct to SRP in non-surgical management of chronic periodontitis.
\end{abstract}

Keywords: Chronic periodontitis, scaling, Root planing, Subgingival irrigation, Ozonated oil, Chlorhexidine, Non-surgical therapy.

\section{Introduction}

Periodontitis is a chronic infection involving destruction of the tooth-supporting apparatus, including the periodontal ligament and alveolar socket support of the teeth. Periodontal disease initiation is by a local accumulation of bacteria and their metabolic products leading to apical migration of the junctional epithelium along the root surface, deepening the gingival crevice to produce periodontal pockets and associated attachment loss, which is the hallmark lesion of periodontal disease. ${ }^{1}$

The microbiology of periodontal infections is complicated for which numerous bacterial agents have been implicated in their etiology. ${ }^{2,3} P$. gingivalis has been known as a major etiologic agent in the development of adult periodontitis. $P$. gingivalis is a highly adapted pathogen that is armed with a number of putative virulence factors that enable this organism to cause disease. Among such putative virulence factors are fimbriae and lectin-type adhesins, a polysaccharide capsule, lipopolysaccharide, hemagglutination and hemolysing activities, toxic products of metabolism, outer membrane vesicles, and numerous enzymes. ${ }^{4}$

Primary goal of therapy is to arrest disease progression and to resolve inflammation for patients with chronic periodontitis. The aim of the therapy is reducing etiologic factors below the threshold capable of producing breakdown, allowing repair of the affected region at a diseased site. Regeneration of destroyed periodontal structures can be enhanced by specific procedures either through nonsurgical periodontal therapy (scaling and root planning (SRP) and the adjunctive use of chemotherapeutic agent) or surgical therapy. ${ }^{5}$ Unfortunately, in some instances, the complex anatomy of the root and the contours of the lesion may hamper the treatment and prevent sufficient reduction of the bacterial load to make the tooth surface biologically acceptable. ${ }^{6}$ This could be related to the persistence of pathogens in the pocket after treatment or to the production of specific virulence factors by the bacteria interfering with the host defense. In this context, it is evident that antimicrobial agents are of great interest and may be valuable as adjuncts to mechanical therapy. These adjunctive therapies are classified by their route of administration to systemic or local drug delivery. ${ }^{5}$

For local administration of antimicrobial agent one of the methods is subgingival irrigation which is intentional irrigation of a gingival crevice or pocket at the point of delivery directed below the gingival margin. It can be done by various antimicrobial agents such as Chlorhexidine, hydrogen peroxide, stannous fluoride, Boric acid and Tetracycline. Even though they show promising results, these existing antiseptic agents have several shortcomings.

Currently, ozone therapy is widely being used as a modern non-invasive method of treatment. It is a powerful oxidizing agent having a high antimicrobial power against oral pathogens, without resistance development has been 
reported not only for gaseous ozone, ${ }^{7,8}$ but also for ozone in aqueous. ${ }^{9}$

The word ozone derived from Greek 'ozein' (odorant) was first used by Schonbein in 1840 (10) when he subjected oxygen to electrical discharges and noted the odor of electrical matter. It was first used in medicine in 1870 by Landler and as a disinfectant by Dr E. Fisch, a Swiss dentist in 1932 in the form of gas or ozonated water. ${ }^{11}$ Ozone has been shown to possess unique properties and has potential applications to the clinical practice of dentistry. There are numerous known actions of ozone such as antimicrobial, immunostimulating, acceleration of the wound healing rate. The three basic forms of application are ozonated water, ozonated olive oil and gaseous ozone, of which the ozonated olive oil being ideal delivery system as it entraps and releases ozone in a sustained manner.

Ozonated olive oil is pure olive oil that has undergone ozonization using a steady flow of ozone- oxygen mixture in the ratio of $5: 95 \%$ until olive oil transforms from the greenish-coloredliquid status to the whitish gel status. ${ }^{12}$ Numerous studies have found their effectiveness against both Gram-positive and Gram-negative bacteria, viruses and fungi. ${ }^{13}$ In the feild of periodontology, it has mainly been used for gingivitis, periodontits, peri-implantitis, wound healing and prophylaxis.

Chlorhexidine is considered to be the "gold standard" agent for chemical plaque control methods, because it is a broad spectrum antiseptic. However, use of chlorhexidine is discouraged because of its unpleasant taste and undesirable side effects such as tooth staining. ${ }^{14}$

The aim of this study was to assess the clinical and microbiological activity of ozonated oil therapy versus $0.12 \%$ chlorhexidine for the treatment of chronic periodontitis.

\section{Materials and Methods}

Twenty patients attending the OPD of the Department of Periodontics, I.T.S CDSR, Muradnagar were screened for this split-mouth randomized controlled clinical trial. The patients (age group 22-65 years) diagnosed with chronic periodontitis having periodontal probing depth $\geq 4 \mathrm{~mm}$, bleeding on probing (BOP) and minimum one periodontal pocket in at least two different quadrants were selected. Inclusion criteria was patients having no history of systemic disease that may affect periodontium, were non-smokers with at least 20 natural teeth; and had not undergone any periodontal treatment in the last 6 months. Exclusion criteria included pregnant women and those with a history of systemic antibiotic administration within 3 months prior to the study. Patients meeting the inclusion criteria were considered for the treatment and follow-up. Patients who agreed for the treatment were enrolled in the study and signed informed consent form was obtained. Ethical approval was obtained from the institutional ethical committee. The history was taken and complete periodontal examination was performed at baseline. Acrylic stent was prepared. Group 1 (control) SRP + subgingival chlorhexidine application was done and in group 2 (test) ozonated olive oil (DENTOZONE INDIA) [Fig 1.] application with SRP was done. Clinical parameters like Plaque index (PI). Gingival index (GI) and Pocket probing depth (PPD) (using a UNC 15 probe) were evaluated at baseline. Plaque samples collection and microbiological analysis was done to assess the change in level of $P$. gingivalis at baseline. SRP was done using ultrasonic scalers (Suprasson Newtron) and Gracey curettes (HuFriedy) until the root surfaces felt smooth and hard to tactile exploration. In the end of the treatment, the periodontal pocket was irrigated by a blunt-tipped sterile plastic syringe containing $0.12 \%$ chlorhexidine (in the control group) [Fig. $3-5$ ] and ozonated oil (in the test group) using disposable 10 $\mathrm{ml}$ plastic syringe.[Fig. 6-8] The selected teeth were isolated with cotton rolls and thoroughly dried and the oil was applied carefully subgingivally until excess oil was observed from the gingival margin. This procedure was repeated for all teeth to be treated. Excess oil was removed with a cotton roll. Periodontal pack was given and patients were instructed not to eat, drink, or rinse for at least $30 \mathrm{mins}$ and were also instructed to refrain from chewing hard or sticky foods, brushing near the treated site or using interdental aids. Instructions with supragingival brushing at the site of application were given. Oral hygiene instructions were given to all patients. After 21 days, patients were reviewed, clinical parameters were again recorded and plaque samples were collected.

Prior to the scaling and root planing and oil application, at baseline subgingival plaque sample was taken from the deepest periodontal pocket for microbiological analysis. The sampling site was isolated with cotton rolls before taking the sample. The supragingival plaque was removed with a sterile curette and cotton gauze. Subsequently, a subgingival plaque sample was obtained using gracey curette.

Sample were kept in the Transport medium (RTF) [Fig 2] which were first vortexed then inoculated in the culture medium according to the requirement in Enriched and selective medium. For, $\mathrm{Pg}$, Blood Agar as a enriched medium, Brucella agar with hemin and vit $\mathrm{K}$ and (BA) was used. Incubation was done at $37{ }^{\circ} \mathrm{C}$ for 3-4 days in anaerobic jar (strictly anaerobe). Kanamycin Blood Agar as anaerobic selective medium was used. Its same as blood agar only Kanamycin is added which makes it selective medium. Then after completion of incubation the plates were removed and the colony characters of the required organism and also the colony count was done for quantification. Then performed for Grams staining - seen for Gram Negative Coccobacilli. These organisms were confirmed by grams staining and key biochemicalsGlucose, sucrose, cellibiose and arabinose.

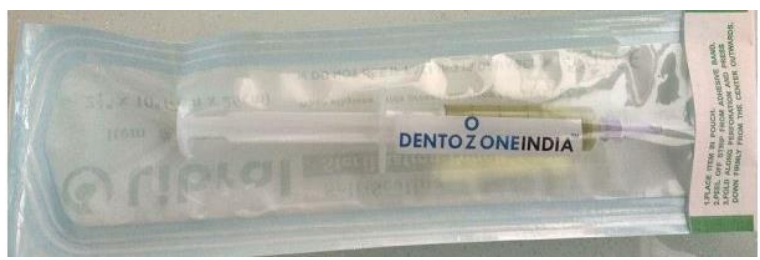

Fig. 1: Ozonated oil (Dentozone India) 


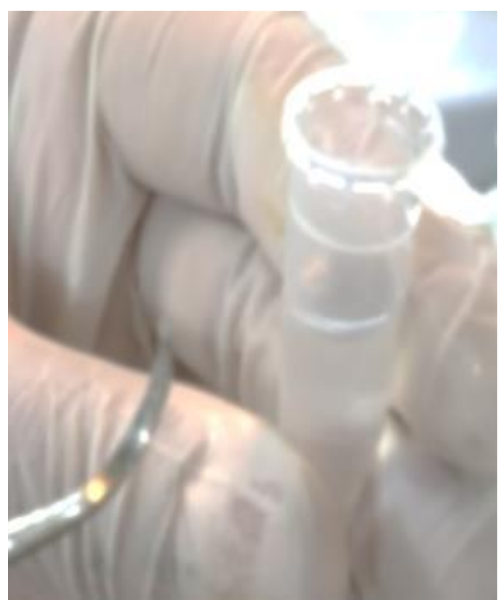

Fig. 2: Plaque sample collection with a curette in a Transport medium

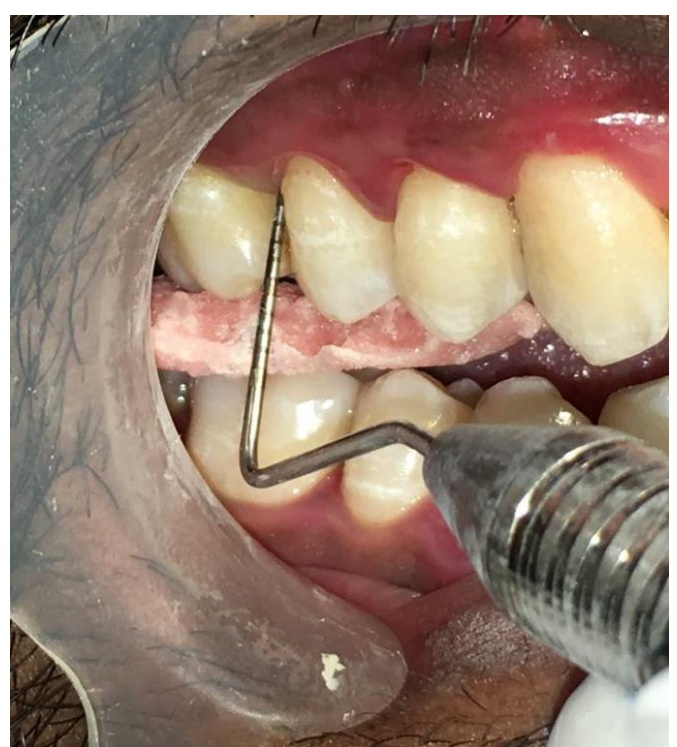

Fig. 3: Pre-operative pocket probing depth measurement in chlorhexidine group

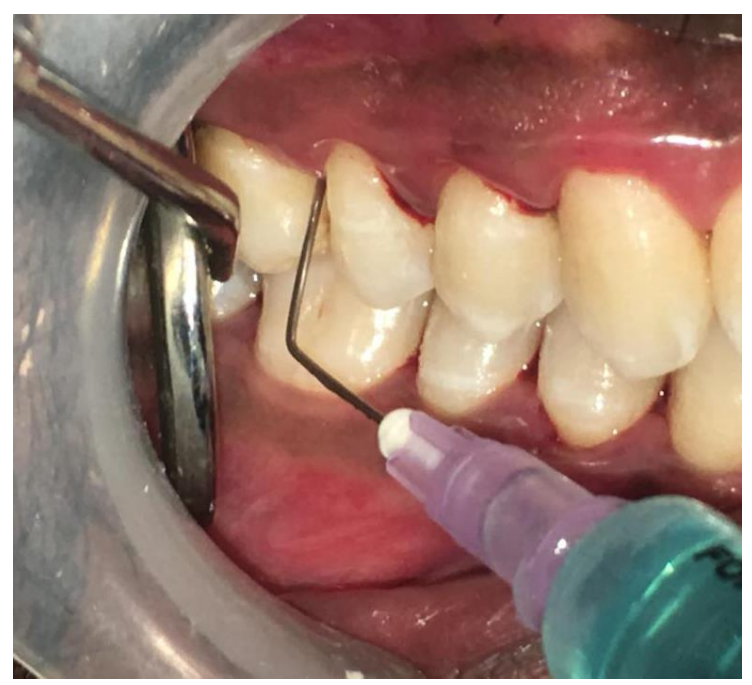

Fig 4: Chlorhexidine irrigation

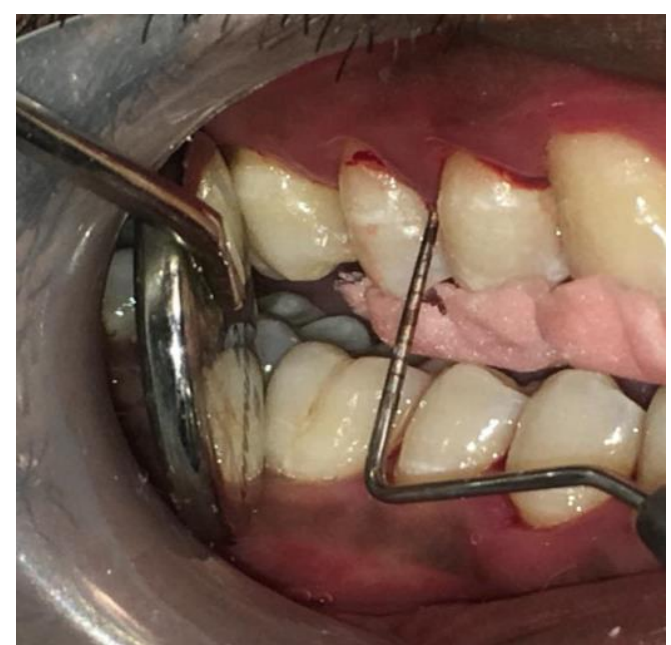

Fig. 5: Measurement of pocket depth after 21 days

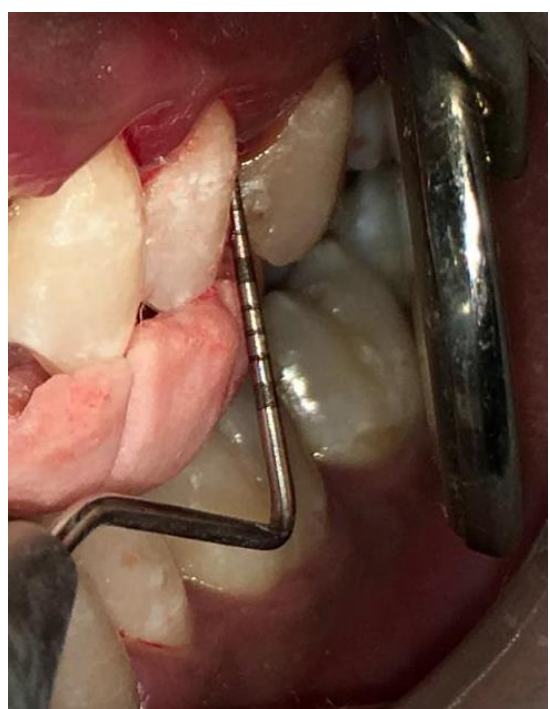

Fig. 6: Pre-operative pocket probing depth in ozonated oil group

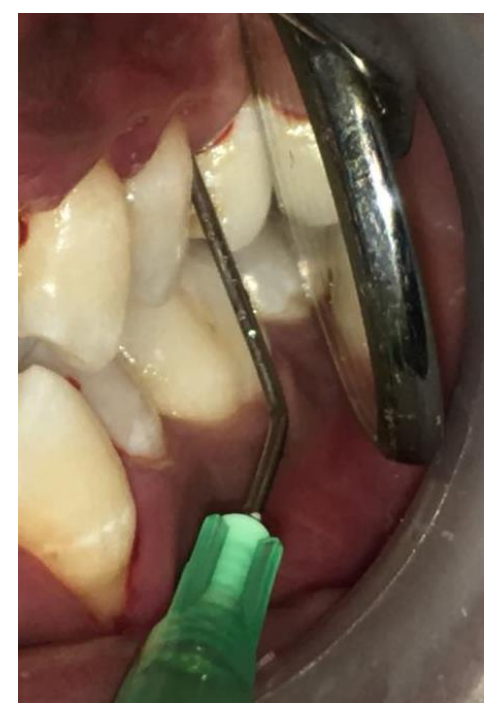

Fig.7: Ozonated oil application 


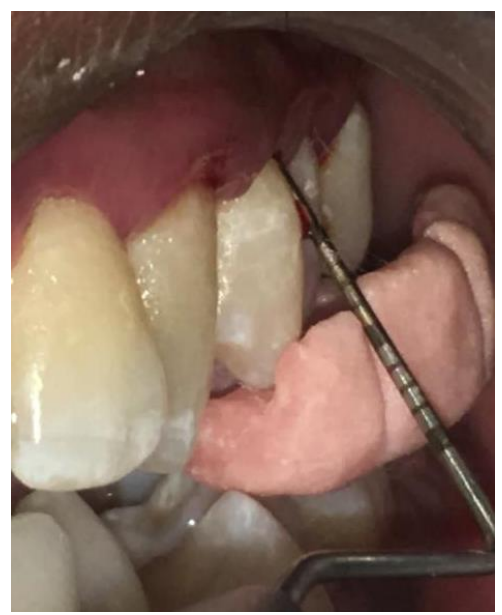

Fig. 8: Measurement of pocket depth after 21 days

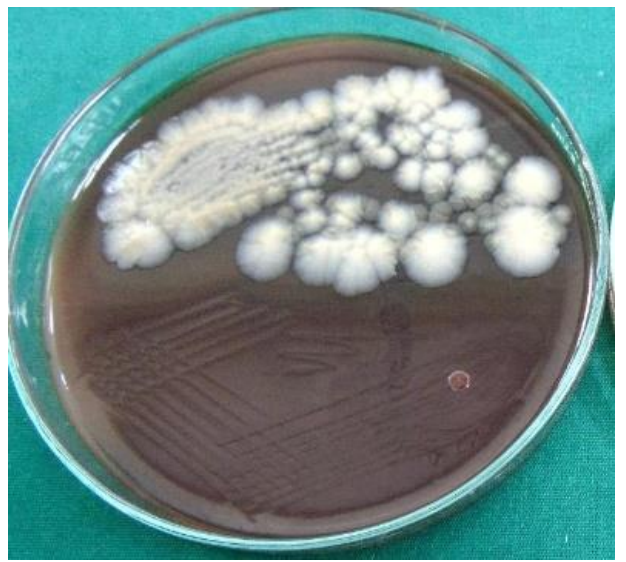

Fig. 9: Kanamycin Blood Agar showing P. gingivalis

\section{Statistical analysis}

The Statistical Analysis was performed using Software (SPSS version 16) for data processing and analysis. The differences in means of the parameters at the baseline and at 21 days between test and control groups were evaluated using an independent t-test. The changes in parameters over time were evaluated using a paired t-test for each group separately, $\mathrm{p}<0.05$ was considered statistically significant.

\section{Result}

In this study, both the groups (test \& control) showed improvement in all the recorded parameters (PI, GI, PPD).No adverse effect were reported by the patients following periodontal therapy. In control group PI, GI\&PPD significantly reduced after 21 days follow-up compared to baseline (Table 1). Similarly, PI, GI \& PPD significantly reduced in Test group also after 21 days follow-up compared to baseline (Table 2). On inter group comparison between test \& control group, there was no statistically significant difference amongst the clinical parameters (PI, GI, PPD) at baseline. However, after 21 days follow-up the test group showed statistically significant improvement in recorded parameters compared to control group (Table 3) (Graph 1.).

For $P$. gingivalis, baseline data shows no significant differences between the two treatment groups. On inter group comparison between test \& control group, there was no statistically significant difference amongst the microbiological count. On intragroup comparison there was significant reduction in P. Gingivalis count from baseline to 21 days.

Table 1: Intra Group Comparison of Clinical and Microbiological Parameters (Test Group)

\begin{tabular}{|c|c|c|c|c|c|}
\hline \multicolumn{1}{|l|}{ Paired Sample Statistics } & N & Mean \pm std. Deviation & Std. Error Mean & p value \\
\hline & & 10 & $2.0750 \pm .624$ & .197 & .296 \\
\hline & PI Baseline & 10 & $.3500 \pm .293$ & .092 & .795 \\
\hline & PI 21 days & 10 & $1.2750 \pm .248$ & .078 & .060 \\
\hline & GI Baseline & 10 & $.1000 \pm .129$ & .040 & .108 \\
\hline & GI 21 days & 10 & $5.60 \pm .699$ & .221 & 1.000 \\
\hline & PPD Baseline & 10 & $3.20 \pm .632$ & .200 & .442 \\
\hline & PPD 21 days & 10 & $2.0579 \pm .153$ & .048 & .511 \\
\hline & P. gingivalis count at baseline & 4 & $1.6990 \pm .000$ & .000 & .044 \\
\hline
\end{tabular}

Table 2: Intra group comparison of clinical and microbiological parameters (Control Group)

\begin{tabular}{|l|l|c|c|c|c|}
\hline Paired Sample Statistics & N & Mean \pm std. Deviation & Std. Error Mean & p value \\
\hline & & 10 & $2.2250 \pm .55840$ & .176 & .296 \\
\hline & PI Baseline & 10 & $.7500 \pm .26352$ & .083 & .795 \\
\hline & PI 21 days & 10 & $1.3750 \pm .37731$ & .119 & .060 \\
\hline & GI Baseline & 10 & $.2250 \pm .21890$ & .069 & .108 \\
\hline & GI 21 days & 10 & $5.60 \pm .699$ & .221 & .000 \\
\hline & PPD Baseline & 10 & $4.60 \pm .699$ & .221 & .442 \\
\hline & PPD 21 days & 10 & $2.0278 \pm .19106$ & .060 & .511 \\
\hline & P. gingivalis count at baseline & 7 & $1.7850 \pm .14689$ & .055 & .044 \\
\hline & P. gingivalis count at 21 days & & &
\end{tabular}


Table 3: Inter group comparison of clinical and Microbiological parameters between test \& control group

\begin{tabular}{|c|c|c|c|c|c|c|}
\hline & Group & $\mathbf{N}$ & Mean $\pm s$ & eviation & Mean & P value \\
\hline PI Baseline & Chlorhexidine & 10 & 2.2250 & \pm .558 & .150 & .578 \\
\hline & Ozonated oil & 10 & 2.0750 & \pm .624 & & \\
\hline PI 21 days & Chlorhexidine & 10 & 1.3750 & \pm .377 & .100 & .493 \\
\hline & Ozonated oil & 10 & 1.2750 & \pm .248 & & \\
\hline GI Baseline & Chlorhexidine & 10 & 5.60 & \pm .699 & .000 & 1.00 \\
\hline & Ozonated oil & 10 & 5.60 & \pm .699 & & \\
\hline GI 21 days & Chlorhexidine & 10 & .7500 & \pm .263 & .400 & .005 \\
\hline & Ozonated oil & 10 & .3500 & \pm .293 & & \\
\hline PPD Baseline & Chlorhexidine & 10 & .2250 & \pm .218 & .125 & .137 \\
\hline & Ozonated oil & 10 & .1000 & \pm .129 & & \\
\hline PPD 21 days & Chlorhexidine & 10 & 4.60 & \pm .699 & 1.400 & .000 \\
\hline & Ozonated oil & 10 & 3.20 & \pm .632 & & \\
\hline$P$. gingivalis count at baseline & Chlorhexidine & 10 & 2.0278 & \pm .191 & .077 & .702 \\
\hline & Ozonated oil & 10 & 2.0579 & \pm .153 & & \\
\hline$P$. gingivaliscount at 21 days & Chlorhexidine & 7 & 1.7850 & \pm .146 & .075 & .282 \\
\hline & Ozonated oil & 4 & 1.6990 & \pm .000 & & \\
\hline
\end{tabular}

Graph 1: Mean Difference of clinical and Microbiological parameters between test \& control group

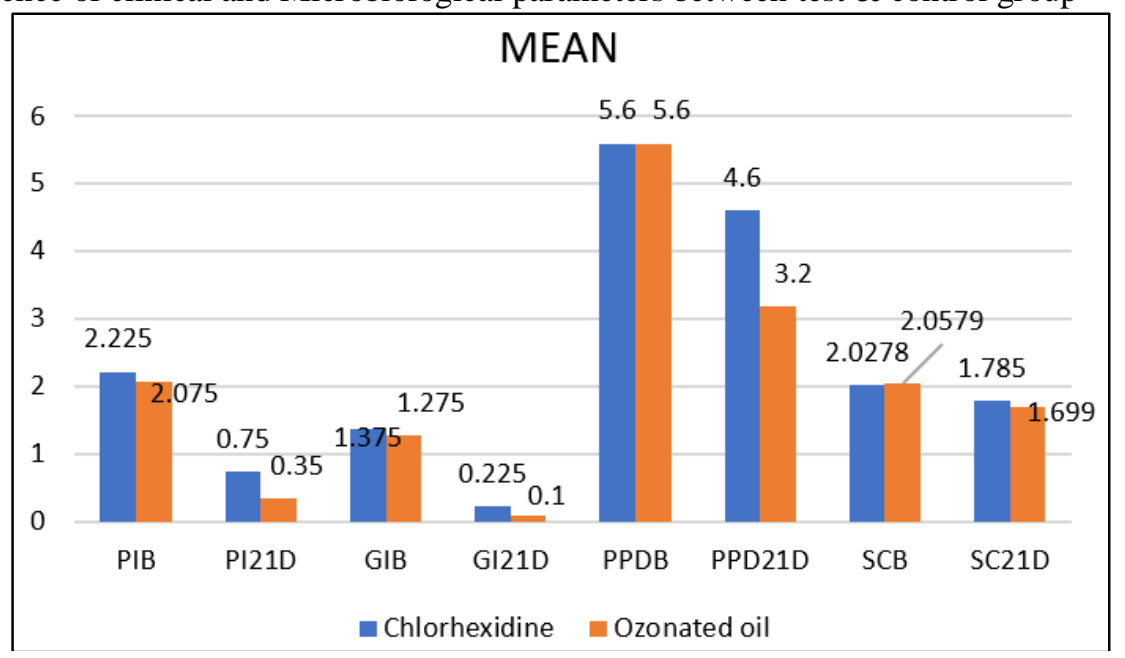

\section{Discussion}

Periodontal disease is a multifactorial disease process in the mouth. The role of microorganisms, host response, in the cause of periodontal disease is well established. The initiation and progression of periodontitis in the subgingival pocketsis because of different bacterial accumulations. The mechanical therapy may help in elimination of the subgingival micro-flora. However its effectiveness is limited by many factors such as concave tooth surfaces, margins of restorations and inaccessibility of periodontal pockets, so mechanical method can also be enhanced by antimicrobial agent. ${ }^{15}$

The periotherapy include, prevention for the non established periodontitis, surgical or the non-surgical remedy for the established periodontitis. ${ }^{16}$ The surgical therapy include excision of the infected tissues and modifying the environment to achieve the best condition for rehabilitating process with meticulous maintenance follow up. ${ }^{17}$ The non-surgical periotherapy is based upon the bacteriological findings and on the susceptibility of the causative microorganisms to the antimicrobial drugs given. ${ }^{18}$

Chlorhexidine has gained popularity as an important oral antiseptic agent and is used as an adjunct to periodontal therapy. It is a broad spectrum antiseptic having antimicrobial effects on Gram-positive as well as Gramnegative bacteria, some viruses, and fungi. ${ }^{19}$ However, because of the undesirable effects such as brownish discoloration of teeth, some restorative materials, the dorsum of tongue and taste disturbance, after prolonged use, several alternatives to chlorhexidine have been investigated. $^{20}$

Ozonated oils are obtained from the chemical reaction between ozone and unsaturated fatty acids of vegetable oils. $^{21,22}$ Due to its excellent curative results, simple 
application, long-term effects, and nontoxic nature they are widely being used.

The aim of the study was to investigate the antimicrobial effectiveness of a commercially available ozonated olive oil (DENTOZONE INDIA) against $P$. gingivalis as a causative organisms intreatment of chronic periodontitis.

There are different ways of delivering ozone, by dissolving in water, it becomes highly unstable and rapidly decomposes through a complex series of chain reactions, so it cannot be stored. ${ }^{23}$ In contrast, when it is dissolved in an oil base, we can measure its life span in yeras. It chemically reacts with oil, and forms long complex molecules. Hence for this study ozonated olive oil gel was chosen over ozonated water because the application of gels stays for longer in the oral cavity, has satisfactory drug penetration, high efficacy and acceptability, this is in addition to the action of omega 3 in olive oil which have potential benefits as a host modulatory agent in the adjunctive management of periodontitis. ${ }^{24}$

Results of the present study revealed that both groups showed improvement in all clinical parameters up to 21 days for PI, GI \& PPD parameters. From the bacteriological point of view results revealed statistically significant reduction of the mean Pg at 21 days for both the groups. There was found to be no significant difference between groups at 21 days for Pg count.

Results of our study was in agreement with an in vitro study by Muller et al. (2007) ${ }^{25}$ reported that there is no additional success in reducing the microbiota on applying ozone. Al Habashneh., et al. ${ }^{26}$ showed that subgingival irrigation with ozonated water as an adjunctive therapy to SRP for chronic periodontitis patients produces no statistically significant difference compared with SRP plus distilled water irrigation.

Study done by Sorokina \& Lukinych (1997) ${ }^{27}$ using subgingival irrigation with ozonized water in combination with professional measures of oral hygiene, plaque formation was found to be reduced due to marked antiinflammatory effects on the periodontium after using irrigation of periodontal pockets with ozone. Kshitish and Laxman $(2010)^{28}$ demonstrated, higher percentage of plaque index (12\%), gingival index (29\%) and bleeding index (26\%) reduction using ozone irrigation. On contrary, Patel et al. $(2012)^{29}$ showed that, the adjunctive use of the Ozonated olive oil gel (OZO) with SRP in treatment of chronic periodontitis resulted in a substantial improvement ( $P$ $<0.001$ ) of clinical parameters as well as microbiological parameters over the time and in comparison to the control groups.

The recolonization of periodontal pocket occurs due to translocation from non-periodontal site by periodontal pathogen that frequently colonizes oral mucosa, tongue dorsum and other oral niches. Also, Hosagi and Duncan ${ }^{30}$ showed that $P$. gingivalis shows protective mechanism to oxidative stress generated by toxic reactive oxygen species (ROS) through up-regulation of anti-oxidant genes resulting in the detoxification of ROS and peroxides. The robustness of this bacterium is not only due to the strong oxidative stress mechanisms that protect its DNA, proteins and lipid layer from oxidative damage, but also because of its superior DNA repair process that accomplishes an efficient and precise removal of deleterious lesions.

The persistence of deep pocket favored recolonization of the putative periodontal pathogens. Accordingly, for assessment of microbial ecological niches surgical management for the pockets is necessary. A more meaningful method is to compare the comprehensive surgical technique comparing the use of ozonated olive oil versus antibiotics in the treatment of deep pocket in chronic periodontitis.

It is worth mentioning that in spite of recolonization of the bacteria in both groups at 21 days, there was a maintained PI, GI \& PPD reduction. The subpopulation of $P$. gingivalis, with both high and low levels of pathogenicity has been suggested to exist among periodontal pathogens harbored by individuals with negligible slight or even severe periodontal destruction. Therefore, there was a lack of association between $P$. gingivalis count in sub gingival plaque samples and severity of clinical signs of the disease.

\section{Conclusion}

Within the limitations of the present study it can be concluded that irrigation with ozonated oil was effective in reducing inflammation and pocket depth in chronic periodontitis patients and can be effectively used as an adjunct to SRP in routine non-surgical periodontal therapy. Ozonated oil as an adjunct is painless and non-invasive and may still find application as an anti-septic in the nonsurgical treatment of periodontal diseases. However, only small to moderate non-significant microbiological improvement of these diseases was found for its adjunctive use in this study; more well-designed studies will be needed to provide insight on the optimal concentration of ozone, duration and frequency of application irrespective of the method of delivery before it can be considered a routine treatment. Ozone is being considered as a promising treatment modality for various dental problems in the near future. But, it has to be kept in mind that presently ozone is used as an adjunct to other conventional treatment modalities so should be used in combination until future research shows benefits in independent usage.

\section{Source of funding}

None.

\section{Conflict of interest}

None.

\section{References}

1. Taylor GBW and Graves D. Periodontal disease and overall health: a clinician's guide. $2^{\text {nd }}$ Edition. Professional Audience Communications, 2010:1-24.

2. Al-Hebshi N. Subgingival periodontal pathogens associated with chronic periodontitis in Yemenis. BMC Oral Health, 2014: 13. 
3. Bonito A. Effectiveness of antimicrobial adjuncts to scaling and root-planing therapy for periodontitis. Evidence Report/Technology Assessment, 2004:1-4.

4. Genco C. Role of gingipains R in the pathogenesis of Porphyromonas gingivalis-mediated periodontal disease. Clinical Infectious Diseases 1999:456-65.

5. Periodontology AAo. Treatment of Plaque-induced Gingivitis, Chronic Periodontitis, and Other Clinical Conditions. 2004;36.16:1-10.

6. Schwach-Abdellaoui K V-CN and Gurny R. Local delivery of antimicrobial agents for the treatment of periodontal diseases. Eur J Pharmac Biopharmac 2000;50.1:83-99.

7. Huth KC, Quirling S, Maier K, Kamereck K, Alkhayer M, Paschos E, et al. Effectiveness of ozone against endodontopathogenic microorganisms in a root canal biofifilm model. Int Endod J 2009;42:3-13.

8. Baysan A, Lynch E. Effect of ozone on the oral microbiota and clinical severity of primary root caries. Am J Dent 2004;17:5660 .

9. Restaino L, Frampton EW, Hemphill JB, Palnikar P. Effificacy of ozonated water against various food-related microorganisms Appl Environ Microbiol 1995;61:3471-5.

10. Schonbein C.F. On the odour accompanying electricity and on the probability of its dependence on the presence of a New Substance. Philos Mag 1840;17:293- 4.

11. Fish E, inventor. Ophthamlic Ventures, assignee. Apparatus for the production and use of ozone in therapeutics. 1936;2:054:367.

12. Cruz O, Menedez S, Martinez ME, Clavera T. Application of ozonized oil in the treatment of alveolitis. In: 2nd International Symposium on Ozone Applications Havana, Cuba; 1997.

13. Gupta M, Abhishek. Ozone: An emerging prospect in dentistry. Indian J Dent Sci 2012;1:47-50.

14. Jones CG. Chlorhexidine: Is it still the gold standard? Periodontol 2000;1997;15:55-62.

15. Kshitish D, Laxman VK. The use of ozonated water and $0.2 \%$ chlorhexidine in the treatment of periodontitis patients: a clinical and microbiological study. Indian J Dent Res 2010;3:341-8.

16. Miliauskaite A, Selimovic D, Hannig M. Successful management of aggressive periodontitis by regenerative therapy: a 3- year follow-up case report. J Periodontol 2007;78:2043-50.

17. Ramfjord SP, Cafesse RC, Morrison EC, Hill RW, Kerry GS, Appleberry EA, et al. A comparative study of 4 types of periodontal treatment. 5 years observations. J Clin Periodontal 1987;14:445-52.

18. Gulnur E, Gul A, Timo S, Hanne L, Levent K, Haluk B. The effect of adjunctive low dose doxycycline therapy on clinical parameters and gingival crevice, ularfl fluid matrix metalloproteinase-8 levels in chronic periodontitis. $J$ Periodontal 2004;75:106-15.

19. Moshrefi A. Chlorhexidine. J West Soc Periodontol Periodontal Abstr 2002;50:5-9.

20. Gagari E, Kabani S. Adverse effects of mouthwash use. A review. Oral Surg Oral Med Oral Pathol Oral Radiol Endod 1995;80:432-9.

21. Patel PV, Gujjari SK. The morphometrical and histopathological changes which were observed after topical ozone therapy on an exophytic fibrous gingival lesion: A case report. J Clin Diagn Res 2013;7:1239-43.

22. Travagli V, ZanardiI, Valacchi G, Bocci V. Ozone and ozonated oils in skin diseases: A review. Mediators Inflamm 2010;2010:6104-18.

23. Hoigne J, Bader $H$. The role of hydroxyl radical reactions in ozonation processes in aqueous solutions. Water Res 1976;10:377-86.
24. Shin WT, Mirmiran A, Yiacoumi S, Tsouris C. Ozonation using micro bubbles formed by electric fifields. Separat Purif Technol 1999;15:271-82.

25. Mu'ller P, Guggenheim B, Schmidlin PR. Effificacy of gasiform ozone and photodynamic therapy on a multispecies oral biofifilm in vitro. Eur J Oral Sci 2007;115:77-80.

26. Al Habashneh R. "Ozone as an adjunct to conventional nonsurgical therapy in chronic periodontitis: a randomized controlled clinical trial". J Periodontal Res 2015;50.1:37-43.

27. Sorokina S, Lukinych L. Ozone therapy as a part of a complex treatment of a paradontium disease. The medical academy of Nizhni Novgorod, Russia. In: Source: 2nd International Symposium on Ozone Applications, Havana, Cuba; 1997;24-6.

28. Kshitish D, Laxman VK. The use of ozonated water and $0.2 \%$ chlorhexidine in the treatment of periodontitis patients: a clinical and microbiological study. Indian J Dent Res 2010;3:341e8.

29. Patel PV, Patel A, Kumar S, Holmes JC. Effect of subgingival application of topical ozonated olive oil in the treatment of chronic periodontitis: a randomized, controlled, double blind, clinical and microbiological study. Minerva Stomatol 2012;61(9):381-98.

30. Henry L. Oxidative stress resistance in Porphyromonas gingivalis. Future Microbiol 2012;7(4):497-512.

How to cite the article: Khare M, Jain KS, Sharma N. Comparative evaluation of effects of subgingival application of ozonated olive oil gel versus chlorhexidine in patients with chronic periodontitis: A clinicomicrobiological randomised trial. $J$ Dent Specialities 2019;7(2):95-101. 